\title{
La répression politique sous l'Estado Novo au Portugal et ses effets sur l'opposition estudiantine, des années 1960 à la fin du régime
}

The political repression during the Estado Novo in Portugal and its impact on the student opposition, from the 1960s until the end of the regime

\section{Guya Accornero}

\section{OpenEdition Journals}

\section{Édition électronique}

URL : http://journals.openedition.org/conflits/18664

DOI : $10.4000 /$ conflits. 18664

ISSN : $1777-5345$

\section{Éditeur :}

CECLS - Centre d'études sur les conflits - Liberté et sécurité, L'Harmattan

\section{Édition imprimée}

Date de publication : 15 juin 2013

Pagination : 93-112

ISBN : 978-2-343-01116-5

ISSN : $1157-996 \mathrm{X}$

\section{Référence électronique}

Guya Accornero, « La répression politique sous l'Estado Novo au Portugal et ses effets sur l'opposition estudiantine, des années 1960 à la fin du régime », Cultures \& Conflits [En ligne], 89 | printemps 2013, mis en ligne le 15 juin 2014, consulté le 31 mars 2021. URL : http://journals.openedition.org/conflits/ 18664 ; DOI : https://doi.org/10.4000/conflits.18664 


\section{La répression politique sous I'Estado Novo au Portugal et ses effets sur l'opposition estudiantine, des années 1960 à la fin du régime}

\section{GuYa ACCORNERO}

Guya Accornero détient un doctorat de sciences sociales de l'Université de Lisbonne et est actuellement chercheur à l'Instituto Universitário de Lisboa (ISCTE-IUL), au Centro de Investigação e Estudos de Sociologia (CIES-IUL), Portugal. Elle est l'auteur de "La rivoluzione prima della rivoluzione " (paru dans Storia e Problemi Contemporanei, 2010), "Contentious politics and student dissent in the twilight of the Portuguese dictatorship: analysis of a protest cycle" (paru dans Democratization en 2012) et de la monographie "Jovens efervescentes. A oposição estudantil ao Estado Novo (1956-1974) » (Lisbonne, ICS, à paraitre en 2013).

Cet article analyse les effets des politiques répressives du régime autoritaire portugais de l'Estado Novo (1933-1974), en se concentrant surtout sur les 10 dernières années de ce régime, entre 1965 et 1974. Cette période nous permet de suivre la naissance d'une gauche «à la gauche » du Parti Communiste Portuguais (PCP, Partido Comunista Português) ${ }^{1}$ qui se manifeste a cette

1. Le PCP, créé en 1921, a été la principale force d'opposition de l'Estado Novo pendant toute sa durée et, jusqu'en 1964, quand d'autres partis de la nouvelle gauche commencent à apparaittre, il a aussi été le seul parti politique organisé dans le camp de l'opposition. Il faut en effet rappeler que le Parti socialiste (PS, Partido Socialista) fut créé seulement en 1973, même si les socialistes, comme Mário Soares, étaient déjà actifs dans des organisations plus ou moins structurées. Vers le milieu des années 1960, le PCP commence à être critiqué par les différents groupes maoïstes, surtout pour sa position considérée comme modérée sur la guerre coloniale - face à laquelle il défendait une stratégie entriste, dans le but d'infiltrer l'armée et donc contraire à la désertion - et sur la façon de mener la lutte contre le régime. Le PCP soutenait par exemple l'exigence de profiter des élections, même si elles n'étaient pas démocratiques, comme moment de mobilisations et de construction d'un « espace de légitimité ». De manière générale, pour les groupes les plus radicaux qui appelaient les citoyens à boycotter la « farce électorale », cette attitude du PCP avait pour seule conséquence de légitimer le régime luimême. La base du PCP était très variée et le parti très influent aussi bien dans le milieu ouvrier, que chez les salariés ruraux de l'Alentejo et les étudiants. Si, à partir de la moitié et surtout à la fin des années 1960, il commence à perdre de l'influence dans le milieu estudian- 
période, surtout parmi les militants estudiantins, et ses liens avec les politiques répressives, surtout pénitentiaires. Suivre les trajectoires des militants ${ }^{2}$ durant cette période permet de regarder ce qui se passe au moment où le régime, ses institutions répressives, tombent.

L'Estado Novo portugais a été un régime autoritaire à tendance totalitaire né pendant l'époque des fascismes européens, mais qui a survécu à la chute de Mussolini et d'Hitler, jusqu'en 1974. En 1926, la première république parlementaire, fondée en 1911, avait été renversée par un coup d'État qui avait instauré une dictature militaire. Ministre des Finances de cette dictature depuis 1928, António de Oliveira Salazar est nommé Président du Conseil en 1932 et, en 1933, par un vote plébiscitaire en faveur de la nouvelle Constitution, il fonde l'Estado Novo. Il devient alors le chef absolu de ce régime jusqu'en 1968, quand il est remplacé, pour des raisons de santé, par Marcelo Caetano. L'Estado Novo s'inspirait surtout du fascisme italien, mais avec des spécificités substantielles, comme la forte inspiration de la doctrine sociale de l’Église catholique et l'absence de politique expansionniste, puisque le Portugal possédait déjà un grand empire colonial. L'Estado Novo a par contre adopté les principes du corporatisme fasciste, aussi bien sur le plan social et économique, que pour la gestion de l'ordre public et le contrôle des dissidents politiques ${ }^{3}$.

L'objectif de l'article est de montrer en quoi l'étude de cas de l'Estado Novo - et en particulier sa chute - permet d'éclairer les effets de la répression

tin, il reste très puissant parmi les ouvriers et en Alentejo où il continue d'être encore aujourd'hui la principale force politique.

2. Comme l'explique Florence Joshua, le concept de «trajectoires militantes » se situe dans un cadre d'analyse d'inspiration interactionniste, selon lequel : "L'action est appréhendée de manière processuelle, comme la résultante de l'interaction entre une histoire individuelle et un contexte structurel. La notion insiste aussi sur le rôle des différents acteurs qui interagissent autour de la trajectoire [...] Dans cette acception, la notion de trajectoire invite aussi à considérer le militantisme à la fois comme phénomène collectif et comme activité sociale individuelle, en s'intéressant aussi bien à l'acteur (et à la façon dont il gère sa trajectoire d'engagement) qu'au groupement militant ». Voir Joshua F., « Les conditions de (re)production de la LCR. L'approche par les trajectoires militantes ", in Haegel F. (ed.), Partis politiques et système partisan en France, Paris, Presses de Sciences Po, 2007, pp. 25-67. Proche de celle de «trajectoire ", mais plus centrée sur le parcours du militant lui-même et sur sa biographie, est la notion de "carrière " qui, " appliquée à l'engagement politique $[. .$.$] permet de comprendre$ comment, à chaque étape de la biographie, les attitudes et comportements sont déterminés par les attitudes et comportements passés et conditionnent à leur tour le champ des possibles à venir, resituant ainsi les périodes d'engagement dans l'ensemble du cycle de vie », Fillieule O., "Carrière Militante », in Fillieule O., Mathieu L et Péchu C. (eds.), Dictionnaire des mouvements sociaux, Paris, Presses de Sciences Po, 2009, pp. 85-94.

3. Paolo Ungari, historien italien du droit, a mis en évidence que l'un des aspects qui distingue l'essence de la codification du droit sous les régimes autoritaires, surtout fascistes, est la tentative de résoudre les conflits et les clivages sociaux par la voie normative, c'est-à-dire le fait de " considérer la loi comme source de structure de la société ». Voir Ungari P., Alfredo Rocco e l'ideologia giuridica del fascismo, Brescia, Morcellina, 1963, p. 67. Pour cet auteur, cela revient à considérer la technique juridique comme " peu technique et très idéologique " pour en faire, au bout du compte, un instrument de violence. Ces considérations peuvent aussi être utiles pour analyser la façon dont l'Estado Novo portugais abordait le contrôle du conflit social et de l'opposition politique. 
sur la politique contestataire, les organisations et les individus, ainsi que les conséquences des politiques répressives et pénitentiaires des régimes autoritaires. L'hypothèse ici est que la répression, dans le cas de l'Estado Novo, a contribué à la (re)constitution même du champ politique des réprimés ${ }^{4}$. Ce processus ne peut pas être réduit au simple binôme répression/radicalisation. Il doit plutôt être vu, en premier lieu, comme un mécanisme par lequel la répression a pu avoir comme effet de «délocaliser » la mobilisation d'un espace à un autre et, par conséquent, de contribuer à sa diffusion. Cela est mis en évidence par l'étude du milieu estudiantin. En outre, comme dans le cas des prisons politiques, la répression a intensifié le processus de socialisation politique. Enfin, en fermant la plupart des sorties possibles de l'engagement politique (par exemple en rendant difficile la poursuite des études et des carrières professionnelles, ou en forçant l'entrée en clandestinité) et en empêchant ainsi l'insertion des militants dans d'autres réseaux sociaux, la répression a contribué à une « radicalisation du militantisme » 5 .

Après avoir présenté une problématisation des mécanismes répressifs à l'œuvre dans le milieu universitaire et ses effets de diffusion de la mobilisation, nous analyserons le processus de socialisation politique au sein des prisons politiques dans le cadre d'une étude de cas, celui d'un militant étudiant, José Luis Saldanha Sanches ${ }^{6}$, l'un des étudiants qui passa le plus de temps en pri-

4. Bourdieu voit la constitution du champ politique comme comme une sorte d' « initiation, avec ses épreuves et ses rites de passages, qui tendent à inculquer la maîtrise pratique de la logique immanente du champ politique et à imposer une soumission de fait aux valeurs, aux hiérarchies et aux censures inhérentes à ce champ ", Bourdieu P., «La représentation politique. Éléments pour une théorie du champ politique ", Actes de la recherche en sciences sociales, 1981, 36-37, p. 6. Le concept de " champ militant » peut aussi aider à expliquer ce processus de «perpétuation » du militantisme, qui devient lui-même un élément fondamental de l'attitude résistante. Le seul fait d'être militant, dans ce contexte autoritaire, devient alors un acte de résistance, parfois indépendamment de l'idéologie partisane à la base de l'engagement (même s'il est évident qu'on se trouve toujours dans un horizon idéologique marqué par le marxisme-léninisme). Selon Cécile Péchu, le champ militant «s'oppose au champ partisan en ce sens que l'illusion qui est propre au premier, la recherche de l'autorité politique légitime, constitue dans le second un repoussoir. Comme le champ artistique, qui s'est constitué en champ indépendant en rejetant la logique économique de la recherche du profit et en proclamant la logique de l'art pour l'art, ce qui constitue en quelque sorte son exact inverse, le champ militant rejette la logique de recherche de l'autorité politique légitime du champ partisan en proclamant une logique du militantisme pour le militantisme, ce qu'exprime bien l'idée essentielle de mise en place de contre-pouvoirs ", Péchu C., "Les générations militantes à Droit Au Logement et l'autonomisation d'un champ militant ", Revue française de science politique, 2001, 51, 1-2, p.73.

5. Nous entendons par « radicalisation du militantisme » un processus relatif tant à la forme de l'engagement qu'à son idéologie, qui tend à isoler les individus, en les éloignant notamment de leurs familles, leur travail, tout en les intégrant de plus en plus fortement dans des réseaux militants. Ce processus s'opère, dans le cadre de cet article, en réaction à la répression exercée par le régime. Au moment où le régime chute, l'investissement dans d'autres réseaux redevient possible, et cela a pour conséquence, dans bien des cas, l'abandon des activités militantes.

6. José Luís Saldanha Sanches (1944-2010) a été dirigeant du mouvement estudiantin contre l'Estado Novo. Il commence sa carrière militante dans le PCP, pour passer après au maoïsme. Il quitte le militantisme direct peu après la chute du régime, pour se consacrer à la carrière universitaire, dans le domaine du droit fiscal, en devenant le fiscaliste portugais le plus connu. 
son dans la dernière période du régime. Après avoir passé la majeure partie de sa « carrière militante» 7 en détention, Saldanha Sanches renoncera à l'activisme peu après la chute du régime, quand l'ouverture du système politique lui permet aussi de développer d'autres activités et d'autres relations. Ceci rejoint, en suivant une approche interactionniste, le point de vue d'Olivier Fillieule : « le coût psychique ou matériel de la défection, et donc sa probabilité, sont redevables de plusieurs facteurs parmi lesquels on mentionnera l'étendue des sacrifices consentis pour entrer dans le groupe [...], la socialisation plus ou moins forte au sein du groupe, qui se traduit notamment par le renforcement de l'attachement émotionnel, lequel varie en fonction du degré de renonciation aux relations sociales extérieures au groupe ${ }^{8}$ ». Ce n'est en effet qu'après la chute du régime et la sortie de prison que Sanches arrive à se désengager : ce sont les liens établis en prison qui ont été décisifs pour expliquer son engagement.

Nous pouvons donc avancer que la transition vers la démocratie au Portugal n'a pas apporté uniquement la possibilité de participation institutionnelle. Elle a aussi permis aux militants de l'opposition portugaise à l'Estado Novo de renoncer au militantisme et de commencer d'autres activités, en ayant la possibilité d'établir des relations sociales extérieures à celles qui étaient à la base de l'engagement politique.

Le matériel empirique utilisé dans le cadre de cet article est constitué d'entretiens avec des militants ou des ex-militants, ainsi que de sources de presse et de sources officielles, comme le Diário da República ${ }^{9}$, les archives du Ministère de l'Intérieur et les archives de la police politique. Notre démarche s'inspire d'une approche interactionniste, avec une attention spéciale portée au rôle des réseaux sociaux dans lesquels le militant est inséré et la fonction de ces réseaux vis-à-vis de la répression politique. Nous montrerons ainsi ce que ces positions permettent de comprendre de la répression et de ses effets sous l'Estado Novo et, en retour, en quoi ce cas d'étude enrichit cette perspective de recherche.

\section{De la répression «sur le papier » à la répression à l'œuvre}

On présentera ici les principaux mécanismes répressifs institués par l'Estado Novo à partir de 1933 et leur évolution au long des années. On cherchera ensuite à montrer ces mécanismes à l'œuvre depuis le milieu des années 1960 dans le milieu du mouvement estudiantin.

7. Voir note 2.

8. Fillieule O. et Benanni-Chraïbi M., «Exit, voice, loyalty et bien d'autres choses encore... ", in Fillieule O. et Benanni-Chraibi M. (eds.), Résistances et protestations dans les sociétés musulmanes, Paris, Presses de Sciences Po, 2003, p. 123 et Fillieule O., "Some Elements of an Interactionist Approach to Political Disengagement”, Social Movements Studies, 2010, vol. 9, $\mathrm{n}^{\circ} 1$, p. 2.

9. Le Bulletin officiel du Parlement portugais. 
Le processus d'autoritarisation de l'État portugais à partir de 1933 : la codification du crime politique et l'institutionnalisation de la répression

Comme mentionné précédemment, l'Estado Novo naît officiellement en 1933, après le vote plébiscitaire de la nouvelle Constitution. La phase postconstitutionnelle est inaugurée dès 1933 par la création sur ordre direct de Salazar de la police politique, la PVDE (Policia de Vigilância e Defesa do Estado, Police de surveillance et de défense de l'État ${ }^{10}$ ), surtout chargée du contrôle du crime politique, et fortement inspirée par l'OVRA, la police secrète du fascisme italien ${ }^{11}$. En 1945, la police politique est rebaptisée Police internationale et de défense de l'État (Polícia Internacional e de Defensa do Estado-PIDE). Le but de ce changement était surtout de montrer une façade plus proche de celle des services secrets des démocraties occidentales, dans la mesure où, après la Deuxième Guerre Mondiale et la chute des fascismes européens et dans la perspective d'adhésion du Portugal à l'OTAN en 1949, il était très difficile de défendre le maintien d'une police politique ${ }^{12}$.

Toujours en 1933, est publié un texte considéré comme un acte fondateur pour la systématisation de la répression politique : le décret-loi $23.203 \mathrm{du} 6$ novembre. Ce texte définit les crimes politiques comme toute propagande, incitation ou provocation par tout moyen des principes fondamentaux de la société. Il détermine aussi que ces crimes relèvent de la compétence d'un tribunal militaire ${ }^{13}$, que la peine d'emprisonnement doit être purgée dans des prisons spéciales et établit la peine d'exil pour certains condamnés considérés plus dangereux.

En 1934, le premier camp de concentration est ouvert près de l'embouchure du fleuve Cunene, en Angola. Mais le camp de concentration le plus important fut celui de Tarrafal, au Cap-Vert, institué par le décret-loi 26.539 du 23 avril 1936, dont l'article 2 stipulait que le camp était destiné aux «prisonniers politiques et sociaux condamnés à l'exil, ou à ceux qui sont détenus dans d'autres établissements carcéraux mais se montrent réfractaires à la discipline ou sont pernicieux pour les autres détenus $» 14$.

10. Voir Pimentel I., A história da PIDE, Lisboa, Círculo de Leitores, 2007.

11. Voir ibidem ; Canali M., Le spie del regime, Bologna, Il Mulino, 2004 et Ivani M., Esportare il fascismo. Collaborazione di polizia e diplomazia culturale tra Italia fascista e Portogallo di Salazar (1928-1945), Bologna, Clueb, 2009. La signification de ce sigle n’a jamais été expliquée, les plus accréditées sont les suivantes : Opera Volontaria per la Repressione dell'Antifascismo (CEuvre Volontaire pour la Répression de l'Antifascisme), Organizzazione di Vigilanza e Repressione dell'Antifascismo (Organisation pour la Vigilance et la Défense de l'Antifascisme) et Organo di Vigilanza dei Reati Antistatali (Organe de Vigilance des Délits contre l'État).

12. Voir Pimentel, A bistória da PIDE, op. cit.

13. Voir Rosas F. (ed.), Tribunais políticos.Tribunais militares especiais e tribunais plenários durante a ditadura e o Estado Novo, Lisboa, Círculo de Leitores, 2009.

14. Toutes les traductions des citations ont été réalisées par l'auteure. 
Le régime instituait aussi des établissements pénitentiaires réservés aux prisonniers politiques : la prison de Aljube, à Lisbonne, qui sera fermée en 1969, la prison de Caxias, entre Lisbonne et la ville de Cascais, la prison de la PVDE à Porto et, enfin, la prison de Peniche, dans la ville maritime du même nom située au nord de Lisbonne, destinée uniquement aux hommes et, surtout, à purger les medidas de segurança (peines de sûreté), c'est-à-dire un système de prolongation des peines, par périodes de trois ans successives, jusqu'à ce que «le prisonnier se montre capable de mener une vie honnête ${ }^{15}$ ». Ce qui fut construit en réalité, c’est tout un système spécial destiné à la définition, au contrôle et à la répression du crime politique, qui devait suivre un chemin complètement différent du crime de droit commun. Par contre, bien que les prisons fussent réservées aux prisonniers politiques, des criminels de droit commun y étaient aussi enfermés, en général pour s'occuper de la cuisine et de l'entretien de l'établissement.

En 1936, surtout à cause de la nécessité de canaliser les âmes les plus radicales à la base du régime (comme par exemple le courant de l'Integralismo Lusitano d'inspiration maurrassienne) et d'accroître la vigilance face à la guerre civile espagnole, Salazar institue la Légion portugaise (Legião Portuguesa - LP), une milice volontaire ayant pour mission de « défendre le patrimoine spirituel de la Nation et combattre la menace communiste et anarchiste 16 ».

Enfin, on peut citer les autres polices, qui existaient déjà avant l'instauration même du régime et qui étaient aussi les principales forces de répression politique sous la République parlementaire : la Police Judiciaire (Polícia Judiciária - PJ) sous la direction du Ministère de la Justice, la Police de Sûreté Publique (Polícia de Segurança Pública - PSP), rattachée au Ministère de l'intérieur et la Garde nationale républicaine (Guarda Nacional Republicana GNR), un corps dépendant du Ministère de la défense et du Ministère de l'intérieur, homologue des carabinieri italiens et de la gendarmerie nationale française. La PSP et la GNR étaient des corps de police fondamentaux pour la répression des mouvements sociaux, la première dans les milieux urbains et la deuxième surtout dans les milieux ruraux.

Le mode d'action du contrôle de l'ordre public changea beaucoup au fil $\mathrm{du}$ temps et se perfectionna surtout entre 1958 et 1962 . Ces quatre années

15. Article 117, décret-loi $\mathrm{n}^{\circ} 26.643$.

16. Décret-loi no 27 058, du 30 septembre 1936. Même si l'inspiration de cette milice était clairement l'italienne Milizia Volontaria per la Sicurezza Nazionale (MVSN, Milice volontaire pour la sécurité nationale, les tristement célèbres camice nere), et en partie l'allemande Sturmabteilung (SA, Bataillon d'Assaut), la Legião Portuguesa ne sera pas une milice partisane comme la MVSN ou la SA, mais un organe de l'État, dépendant des ministères de l'Intérieur et, en cas de crise, de celui de la Guerre. Cette différence est aussi justifiée par le fait que, contrairement à l'Italie et à l'Allemagne, la milice n'a pas été au Portugal un instrument pour affirmer le régime, mais a été créée après. Voir, Rodrigues L.N., A Legião Portuguesa: a milícia do Estado Novo (1936-1944), Lisboa, Editorial Estampa, 1996. 
furent l'une des périodes les plus problématiques pour le régime, à cause des grands conflits sociaux qui touchaient les secteurs les plus divers de la société. Ceci débuta pendant la campagne électorale pour les élections présidentielles de 1958 qui fut marquée par la candidature, contre le candidat du régime, du Général Humberto Delgado qui promettait une ouverture du système politique et la destitution de Salazar et qui fut soutenu par de grandes mobilisations à travers tout le pays. Dans un système de consultation électorale non démocratique, Humberto Delgado perdit évidemment les élections, mais sa candidature n'était que le premier signe d'une vague de contestation qui atteindrait son sommet avec la crise ouvrière et estudiantine de 1962, un an après le début de la guerre coloniale contre les mouvements indépendantistes au Mozambique, en Angola, en Guinée et au Cap-Vert.

À partir de ce moment, on assiste à une augmentation importante des interventions de la PSP au détriment de la GNR, liée surtout à l'urbanisation du conflit (en raison de la forte diminution des travailleurs du secteur primaire à partir de 1960 et de la correspondante augmentation de ceux du secteur secondaire et, à partir de la fin des années 1960, du tertiaire), mais aussi à l'augmentation du nombre d'étudiants dans les universités ${ }^{17}$. La PSP devient, jusqu'à la fin du régime, le vrai bras exécutif de la PIDE - même si elle n'est pas toujours en accord avec elle - et son rôle dans la répression des manifestations de rue sera crucial ${ }^{18}$.

D'autres transformations eurent lieu ensuite sous le «Marcelismo», c'està-dire la dernière période de la vie de l'Estado Novo qui s'ouvre en 1968 avec le remplacement de Salazar par Marcelo Caetano à la tête du gouvernement. Si la réforme de la PIDE en 1969 (qui devient la Direction générale de la sûreté Direcção Geral de Segurança-DGS), ne change guère les compétences de cette police, les réformes du Code pénal et du Code de procédure pénale, qui interviennent en 1972 après la révision de la Constitution de 1971, eurent plus d'impact. Les principaux changements concernaient la suppression des mesures de sûreté (en particulier pour les crimes politiques) qui eut pour effet immédiat, comme on le verra, de permettre à des nombreux prisonniers politiques de sortir de prison.

17. Voir Barreto A. (ed.), A Situação Social Em Portugal, 1960-1999, Lisboa, Imprensa de Ciências Sociais, 2000. En 1958, les interventions d'ordre public de la PSP ont représenté $4 \%$ sur le total des interventions réalisées par l'ensemble des forces de l'ordre, alors que celles de la GNR ont été de $15 \%$. En 1962, les interventions de la PSP s'élèvent à $43 \%$, tandis que celles de la GNR descendent à $6 \%$ et, en 1972, cette proportion s'établit à $63 \%$ pour la PSP contre $9 \%$ pour la GNR (Données rassemblées par l'auteure dans Registro da Correspondência Recebida, années 1956-1974, Arquivo do Ministério do Interior, Institutos dos Arquivos Nacionais Torre do Tombo, Lisbonne). Les principales villes où se concentrait le conflit étaient Lisbonne et Setúbal (pour le conflit ouvrier), et Lisbonne, Coimbra et Porto pour le conflit estudiantin.

18. En effet, la PIDE avait uniquement des fonctions d'investigation et réalisait des arrestations ponctuelles. Même si la PSP aussi pouvait réaliser des investigations ou procéder à des arrestations ponctuelles, elle était surtout destinée au contrôle de l’ordre public directement « dans la place » et c'est dans ce contexte que la plupart de ses arrestations s'opéraient. 
Le système répressif de l'Estado Novo était basé sur la violence et sur le contrôle et la persécution presque totale des adversaires politiques. Toutefois, quand on passe de la simple description des instruments répressifs à l'analyse de leur mise en œuvre, on s'aperçoit que ce système comporte plusieurs facettes, dont certaines se contredisent. Le principal objectif de ce système étant celui d'éviter le conflit, vrai cauchemar pour l'idéologie corporatiste, on trouve aussi, à côté du recours indiscriminé à la force, des mécanismes de répression sélective, des techniques de désescalade, des tentatives de dialogue avec les adversaires plus modérés ou d'infiltration, ainsi que la prise en considération des effets sur l'opinion publique des excès répressifs. En plus de ces contradictions, en réalité seulement apparentes, on en trouve aussi d'autres moins « rationnelles ", issues par exemple des divisions entre les différentes polices, ou d'erreurs stratégiques.

Le cas de la répression du mouvement estudiantin qui se développe autour de l'université de Coimbra constitue un excellent exemple de ce type de contradictions, non seulement parce qu'il est très bien documenté par les archives policières, mais aussi parce qu'il présente très clairement les dynamiques dont on a parlé jusqu'ici. En 1969, l’université de Coimbra connaît une forte agitation, dont l'épisode le plus emblématique est le mouvement de contestation du 17 avril contre le Président de la République, Américo Tomás, pendant l'inauguration des nouveaux bâtiments de la Faculté de mathématiques. Cet événement n'était pas improvisé, et il intervient après les élections pour la nouvelle direction de l'Association d'étudiants qui s'étaient tenues un mois auparavant et avaient donné la victoire, avec $75 \%$ des votes, à une liste de gauche, la liste du Conseil des républiques (Conselho das republicas).

Ces élections faisaient suite à quatre années pendant lesquelles la direction de l'Association était entre les mains d'une commission administrative nonélue et contrôlée par les autorités. La mobilisation pour la réalisation d'élections libres afin de choisir les représentants des étudiants avait constitué une revendication fondamentale et la crise de 1969 en a été le résultat. Le fait que l'université de Coimbra se trouvait sans organismes légaux effectivement représentatifs avait certes permis une période de calme mais avait aussi stimulé le développement de réseaux alternatifs comme base pour le conflit, plus informels et moins contrôlables par les autorités. Ces réseaux se constituaient surtout autour du circuit des Republicas, les traditionnelles résidences étudiantes de Coimbra, considérées alors par la police comme presque toutes «de gauche 11 .

19. Accornero G., Efervescência estudantil. Estudantes, acção contenciosa e processo político no final do Estado Novo, thèse de doctorat en sociologie historique, Instituto de Ciências Sociais da Universidade de Lisboa, 2010, p.113. 
Même pour ce qui concerne la forte politisation de ces organismes, on trouve un premier contraste entre la parole officielle et l'expression des difficultés rencontrées par la police politique pour gérer la situation. Un rapport de la PIDE/DGS dénonce en effet que le succès des Republicas est aussi provoqué par «le manque d'initiative de la commission administrative » 20 imposée par les autorités. Par ailleurs, la police politique souligne que « des points de contact et des influences [parmi les étudiants] sont compromis par l'inactivité des organismes de droite et par l'inexistence d'une maison estudiantine de droite » 21. La police déplore ainsi que les rares étudiants de droite «n'ont pas leur propre groupe organisé » et « font des distinctions entre droite fasciste, républicaine, corporatiste et démocratique» 22.

Après les événements de contestation du Président de la République en avril 1969, l'association d'étudiants fut aussitôt interdite et les dirigeants fraîchement élus arrêtés. Toutefois, cette mesure, selon la PIDE/DGS, pouvait de nouveau amener des effets contraires, notamment une radicalisation et une diffusion de la protestation, puisque « avec la fermeture de l'association, on a évité les réunions de masse, mais pas l'activité des plus politisés, qui se retrouvent dans les repúblicas ou au dehors de la ville, avec le soutien de fonctionnaires de l'université ou autres travailleurs ${ }^{23}$.

Mais la plupart des critiques de la PIDE/DGS étaient dirigées contre les autres forces de police, surtout la PSP, considérée comme " pas trop raffinée » pour ce qui concerne les techniques de gestion de l'ordre public. Le 25 septembre 1969, une manifestation fut réprimée dans une rue de Coimbra par la PSP, provoquant plusieurs blessés parmi les étudiants qui furent hospitalisés et interrogés à l'hôpital 24. Dans un rapport de la PIDE/DGS, il est indiqué que le 25 septembre a été «transformé en une journée de folklore politique, avec coups de bâton, gaz lacrymogènes, taches de sang sur les trottoirs, étudiants hospitalisés, cris d'assassins contre la PSP et en plus des magasins qui ont fermé pour éviter les dégâts » 25 . Le principal problème était que ces événements avaient touché aussi des civils en marge des manifestations qui avaient ensuite porté plainte à la préfecture.

Le problème de la coordination des forces de l'ordre et du dosage de l'usage de la violence est en effet une constante de cette période. Toujours en 1969, le 25 novembre, à l'occasion d'une manifestation commémorative de la

20. Rapport de la PIDE de Coimbra, 19 septembre 1968, en Instituto dos Arquivos Nacionais -Torre do Tombo (IAN-TT)-Archives de la PIDE/DGS-SC-SR-3529/62, doc.60.

21. Ibidem.

22. Rapport de la PIDE de Coimbra, sans date en IAN/TT-PIDE/DGS-SC-SR-3529/62-3364pasta (PT, enveloppe) 152, doc. 68.

23. Rapport de la PIDE/DGS de Coimbra, septembre 1969, PIDE/DGS-SC-SR-3529/62-3364PT.152, doc. 68.

24. Rapport de la PIDE de Coimbra, 26 septembre 1969, en IAN/TT-PIDE/DGS-SC-SR3529/62-3364-Pt.152, doc. 13.

25. Ibidem. 
«prise de la Bastille » (tomada da bastilha) ${ }^{26}$, un document de la PIDE/DGS souligne que ce jour pouvait donner lieu à une grande agitation estudiantine, à cause de l'arrestation par la PSP d'un étudiant en droit accusé d'incitation à la grève. Le document continue ainsi : « on ne veut pas faire de jugements critiques sur cette action de la PSP, mais notre délégation doit considérer qu'il serait une question de prudence de chercher à faire le moins d'arrestations possibles, parce que les arrestations peuvent créer de situations émotives et accélérer ou réveiller des situations latentes 27 ».

Les conflits entre autorités continuent autour de la question de l'autorisation par le préfet de manifestations le jour de la tomada da bastilha. Selon l'avis des agents de la PIDE/DGS, opposés à l'autorisation, il pouvait y avoir des accidents pendant la commémoration « avec tous les risques d'un malheur pendant la répression, qui susciterait une vive émotion dans tout le pays 28 ». En d'autres occasions, la PIDE/DGS accusait directement la PSP de faire un usage exagéré et grossier de la violence, parfois même par le recours à des grenades « d'autant plus que, par les fenêtres de bâtiments, beaucoup de gens observaient l'action policière 29 ».

Ces types de critiques se poursuivirent au cours des années suivantes, caractérisées par une intensification et surtout une radicalisation de l'activisme estudiantin, mais aussi par un contrôle croissant de la part des forces de l'ordre. Même la présence constante d'agents de la PSP dans les locaux et devant les portes de l'université était vue comme une pratique dangereuse par les agents de la PIDE/DGS. Cette attitude, qui créait une « atmosphère de peur moins propice à l'étude » (d'autant plus que les agents de la PSP dispersaient tous ceux qui se trouvaient au dehors de l'université) et « empêchait la socialisation normale typique des étudiants à la sortie des cours » pouvait stimuler une réaction chez les étudiants jusque-là indifférents ${ }^{30}$.

\section{$\mathrm{La}$ « radicalisation » du militantisme ou le paradoxe autoritaire}

Nous avons précédemment introduit en partie l'argument des effets contradictoires de la répression par rapport aux contradictions des mécanismes répressifs eux-mêmes. Nous allons analyser maintenant plus précisément la façon dont la répression a contribué à la constitution même du champ politique des réprimés, surtout dans le milieu pénitentiaire.

26. C'est ainsi que les étudiants de Coimbra appelaient l'occupation, le 25 novembre 1920, d'une partie du bâtiment universitaire destiné aux réunions d'un organisme culturel considéré élitiste, le «Club dos Lentes », pour revendiquer de meilleures installations universitaires.

27. Rapport de la PIDE de Coimbra, 11 novembre 1969, en IAN/TT-PIDE/DGS-SC-SR3529/62-3364-Pt.153, doc. 431.

28. Rapport de la PIDE de Coimbra, 19 novembre 1969, en IAN/TT-PIDE/DGS-SC-SR3529/62-3364-Pt.153, doc. 352.

29. Rapport de la PIDE/DGS de Coimbra, 26 novembre 1969, en IAN/TT-PIDE/DGS-SC-SR3529/62-3364-Pt153, doc. 318,

30. Rapport de la DGS de Coimbra, 17 février 1971, en IAN/TT-PIDE/DGS-SC/SR-3529/62- 
La répression contre les étudiants pouvait être appliquée de différentes façons, même si l'une n'excluait pas toujours l'autre. Les étudiants responsables des agitations pouvaient être expulsés de l'Université et ces expulsions pouvaient être plus ou moins lourdes : pendant une période limitée ou indéfiniment, d'une seule université ou de toutes les universités du pays. Si les cas d'étudiants expulsés de toutes les universités du pays pour une durée indéfinie ont été très rares, une peine d'expulsion plus légère impliquait tout de même pour les étudiants un retard d'au moins un an dans leurs études. Beaucoup d'activistes estudiantins durent pour cela quitter le pays pour poursuivre leurs études, notamment en France, en Suisse et en Belgique où, souvent, ils poursuivaient aussi leur engagement contre le régime ${ }^{31}$. À partir de 1969 et surtout à cause de la crise de Coimbra, les étudiants subversifs commencèrent à être punis aussi au moyen de la conscription anticipée. À cause de la guerre coloniale, presque tous les jeunes portugais étaient astreints à un service militaire de 2 à 4 ans. Cependant, les étudiants avaient normalement le droit de différer cette obligation. Par le décret-loi 49.099 du 4 juillet 1969, le gouvernement suspendit ce droit dans le cas d'étudiants responsables d'agitations, de sorte que presque tous les leaders étudiants durent interrompre leurs études pour satisfaire à leurs obligations militaires. Même dans ce cas, l'action répressive n’a pas atteint l'objectif visé. Elle a au contraire contribué à délocaliser la mobilisation du milieu universitaire vers le milieu militaire, constituant probablement un point encore plus névralgique ${ }^{32}$.

La façon la plus dure de persécuter les étudiants subversifs était sans conteste l'arrestation. Cependant, dans ce cas aussi, la punition avait rarement pour effet d'éradiquer la mobilisation. La prison politique, la plus haute concrétisation du système répressif, contribua en réalité à intensifier la socialisation politique et à redéfinir les identités politiques. C'est cet aspect que nous allons analyser à présent, en suivant le parcours militant de l'activiste José Luís Saldanha Sanches.

3367-Pt.158, doc. 258.

31. Par exemple, après la crise de 1962, sont expulsés de l'Université de Coimbra au moins 3 étudiants pendant 30 mois, 22 étudiants pendant 24 mois, 9 pendant 18 mois. 23 étudiants de Coimbra sont expulsés de toutes les universités du pays pendant 30 mois (source : Archives de la PIDE/DGS, en IAN/TT-PIDE/DGS-SC-SR-1615/61-3096, doc. 25, 18 décembre 1962). Parmi les étudiants expulsés de toutes les universités du pays après la crise de 1962, il y avait le groupe à l'origine du Mouvement d'Action Révolutionnaire (MAR, Movimento de Acção Revolucionária), qui fut constitué à Genèvre en 1963. Malgré son nom, ce n'était pas une organisation radicale, mais elle incluait des militants issus du Parti Communiste qui se rapprochaient des socialistes.

32. La PIDE/DGS notait par exemple une agitation croissante à l'École d'infanterie de Mafra, où la plupart des leaders de la crise de Coimbra avaient étés amenés. Dans un rapport, cette police soulignait que cela avait permis la désertion de huit étudiants, réfugiés en France, et que le responsable était surtout Celso Cruzeiro, leader estudiantin considéré comme « l'activiste le plus dangereux de cette conscription » (rapport de la PIDE-DGS, 3 novembre 1969, en IAN/TT-PIDE/DGS, SC-SR-3529/62-3364, PT 3, doc. 439). 
La première détention de ce militant commença le 29 d'avril 1964. Il fut arrêté alors qu'il collait des affiches du PCP pour la manifestation du $1^{\mathrm{er}}$ mai. Cette première détention se termina le 10 décembre de la même année, c'est-àdire le jour même de son procès, quand, grâce à la défense de l'avocat Mario Soares, le tribunal le condamna à une peine inférieure de deux mois à celle qu'il avait effectivement déjà purgée. Ruben de Carvalho ${ }^{33}$, à l'époque très proche de lui, affirme que, une fois sorti de prison, Saldanha Sanches retourna à la Faculté de droit et s'engagea encore plus dans le PCP, où étaient débattues les «thèses chinoises ", qui semblaient l'attirer beaucoup. Toujours selon de Carvalho, un mois après sa sortie de prison et à cause des arrestations incessantes d'étudiants, Sanches entra dans la clandestinité et se cacha dans une maison louée par le Parti. Grâce à des aveux obtenus sous la torture, la PIDE le trouva et il fut de nouveau arrêté le 18 décembre 1965.

Ici commence selon nous la détention la plus importante dans le parcours de Sanches. Au moment de son arrivée à la prison de Caxias et pendant son incarcération à Peniche, le 2 novembre 1966, les membres de la cellule centrale des militants du Front d'action populaire 34 (la Frente de Acção Popular FAP), première organisation maoïste portugaise à avoir théorisé la nécessité de la violence révolutionnaire, avaient été arrêtés à la suite des premières actions de lutte armée. Certains militants de la FAP, comme le médecin Rui d'Espiney, avaient déjà été en contact avec Saldanha Sanches, avec qui ils avaient participé à la campagne d'Humberto Delgado et à l'organisation des mouvements étudiants à Lisbonne au début des années 1950. Sanches rencontrera les autres - Saul Nunes, Acacio Barata Lima et le dirigeant de la FAP, Francisco Martins Rodrigues - en prison. Les deux militants avec lesquels il aura le plus de contact sont Saul Nunes et Acacio Barata Lima.

Saul Nunes et Saldanha Sanches se connurent dans la prison de Caxias en 1966. En 1967, le premier fut transféré à la prison de Peniche, où Saldanha Sanches se trouvait déjà depuis quelques mois, et il affirme qu'à ce moment-là ce dernier « montrait déjà des signes d'évolution politique 35 ». Son éloignement du PCP se manifeste à cette période par des épisodes qui se produisent à l'intérieur de la prison et qui, même dans un espace où le contrôle est quasi

33. Entretien avec Ruben de Carvalho, réalisée à l'Instituto de Ciências Sociais, Lisbonne, le 26 octobre 2010.

34. La FAP fut la première organisation maoiste portugaise. Elle fut créée à Paris en 1964 par un ancien dirigeant du PCP, Francisco Martins Rodrigues, à partir d'un groupe formé par, entre autres, Rui d'Espiney, João Pulido Valente, Humberto Belo et Manuel Claro. Peu de temps après, ces militants fondèrent le Comité Marxista Leninista Português (CMLP, Comité marxiste-léniniste portugais). La FAP avait une base assez mélangée, avec des militants des professions hétérogènes, à la différence des organisations maoïstes qui vont apparaître dans le futur, dont la base sera surtout estudiantine, même si elles seront présentes parmi les travailleurs du secteur tertiaire, qui était en grande expansion et, d'une façon bien plus limitée, dans le milieu ouvrier.

35. Entretien avec Saul Nunes, réalisé à l'Instituto de Ciências Sociais, Lisbonne, le 8 octobre 2010. Saul Nunes était jardinier du parc de la Fondation Gulbenkian avant de devenir avocat. Jusqu'en 1964, quand il adhéra à la FAP, il avait été compagnon de route du PCP. 
total, montrent bien la division entre «communistes prosoviétiques » et «communistes prochinois ». Par exemple, Saul Nunes tient toujours à rappeler que, le jour de la mort d'Ho Chi Min le 2 septembre 1969 36, les détenus prochinois organisèrent une manifestation de deuil en respectant une minute de silence, debout, lors du déjeuner, et que Saldanha Sanches participa à cette initiative. Dans un milieu comme celui de la prison politique, caractérisé par des possibilités de communications très réduites, de telles actions étaient très emblématiques. Les commémorations étaient des occasions d'affirmer les différences politiques, la position de chaque détenu et les éventuels changements d'alignement. Acácio Barata Lima raconte que les « clivages dans les comportements politiques 37 » se manifestaient de la façon suivante :

«Les prisonniers liés au PCP célébraient la date de l'instauration de la République portugaise, le 5 octobre, et la date de la Restauration de l'Indépendance, le 1er décembre ${ }^{38}$. Les prisonniers qui n'étaient pas liés au PCP commémoraient l'anniversaire de la Révolution chinoise, le $1{ }^{\mathrm{er}}$ octobre. Mais les prisonniers des deux tendances politiques célébraient aussi bien la Fête du Travail, le $1^{\text {er }}$ mai, que la Révolution russe, le 17 octobre (7 novembre selon le nouveau calendrier). Les commémorations consistaient à mettre un costume et une cravate rouge 39 ».

On peut voir dans ces dynamiques le processus de reconstitution du champ politique réprimé, au moyen d'une « prise de pouvoir interne » par les prisonniers eux-mêmes ${ }^{40}$.

36. Les prisonniers n'avaient pas accès aux informations, les journaux étaient triés et arrivaient toujours en retard de quelques jours. Cependant, les détenus de droit commun, qui étaient incarcérés dans les prisons politiques pour y accomplir des tâches d'entretien, s'efforçaient de transmettre les nouvelles aux détenus politiques, bien que cela fût très difficile à cause de l'interdiction de communiquer.

37. Entretien avec Acacio Barata Lima, réalisé à Porto, le 8 novembre 2010 et emails. Acacio Barata Lima fut militant pendant la campagne Delgado et dirigeant estudiantin à l'Université de Porto entre 1957 et 1962. Il adhéra à la FAP en 1964. Après la chute du régime, il a travaillé comme ingénieur pour le gouvernement de Mozambique, pour retourner ensuite au Portugal et y exercer la même profession.

38. Restauration de l'indépendance du Portugal après la domination espagnole, le 1er décembre 1640.

39. Entretien avec Acacio Barata Lima, op. cit.

40. On observe notamment les mécanismes rituels à la base de l'adhésion au champ (voir note 2), mais aussi l'adhésion au "militantisme pour le militantisme ", comme « mise en place de contre-pouvoirs » que Péchu identifie comme l'élément distinctif du champ militant (Péchu C., "Les générations militantes... », op. cit. De l'autre côté, en acceptant la suggestion de Fillieule et Bennani-Chraibi, on peut suivre la vision de Michel de Certeau et observer les dynamiques internes à la prison politique portugaise comme un processus de prise de parole, où, sous le monothéisme de la domination et de la surveillance panoptique, "survivrait un polythéisme de pratiques disséminées, dominées, mais non pas effacées pas la carrière triomphale de l'une d'entre elles ", De Certeau M., L'invention du quotidien, tome I, Arts de faire, Paris, Gallimard, 1990, p.105; cité in Fillieule O. et Bennani-Chraibi M. (eds.), Résistance et protestations dans les sociétés musulmanes, op. cit., p. 30. 
On peut aussi, comme souligné par Gilda Zwerman et Patricia Stheinhoff dans leur étude sur l'interaction entre État et militants de la nouvelle gauche au Japon et aux États-Unis ${ }^{41}$, y voir la capacité des organisations de militants de se régénérer et développer des stratégies de défiance vis-à-vis de la répression de l'État. Il y aurait ainsi un mouvement social distinct : une mobilisation de résistance contre la répression de l'État, avec ses propres caractéristiques et sa propre trajectoire. Les auteurs expliquent ainsi que l'attention portée sur la résistance, et non sur la victime, rend possible l'examen du pouvoir et de sa capacité d'action ${ }^{42}$.

Lima se souvient que, en 1967, Sanches ne célébrait ni le 1er décembre ni le 5 octobre, mais il célébrait le 17 octobre, manifestant donc une attitude ouvertement dissonante par rapport aux autres militants du PCP. Il suggère aussi qu' « il est probable que sa rupture avec le PCP ait eu lieu dès 196643 ». D’après ces indications, on peut penser que les « conversions politiques » dans le milieu carcéral ne se produisaient pas de manière brusque, mais qu'il pouvait y avoir des périodes, même longues, de superpositions pendant lesquelles les frontières entre les différentes tendances politiques étaient floues. Or, si cela paraît plausible jusqu'à la fin des années 1960, à partir de là les clivages se creusent et les conflits entre les prisonniers de différents courants deviennent plus durs. La rupture entre communistes prosoviétiques et communistes prochinois devient définitive au début des années 1970. À cette époque, la prison de Peniche est secouée par une série de grèves de la faim contre les conditions de détention, auxquelles les détenus du PCP ne participent pas. Cette position contribue à accroître les tensions entre les militants des deux courants et le conflit devient si violent que la direction de la prison est obligée de séparer les prisonniers par étages, en fonction de leur affiliation politique.

Cette situation à la prison de Peniche est rappelée dans une interview radiophonique réalisée le 25 avril 1997 avec Saldanha Sanches et Fernando Rosas, un autre militant qui était passé du PCP au maoisme et qui fut l'un des fondateurs du Mouvement de Reconstruction du Parti du Prolétariat (Movimento de Reconstrução do Partido do Proletariado - MRPP) ${ }^{44}$.

41. Zwerman, G. et Steinhoff, P., "When Activists Ask for Trouble: State-Dissent Interactions and the New Left Cycle of Resistance in the United States and Japan”, in Davenport C., Johnston H. et Mueller C. (eds.), Repression and Mobilization, Minneapoli-London, University of Minnesota Press, 2005.

42. Ibid., pp.88 et 90

43. Entretien avec Acacio Barata Lima, op. cit.

44. Le MRRP est créé en 1970 par un groupe formé surtout d'étudiants, dont certains avaient été militants du PCP, qui avaient été auparavant à la base de la Gauche Démocratique Estudiantine (EDE, Equerda Democrática Estudantil), l'organisation qui avait réalisé la première manifestation contre la guerre du Vietnam au Portugal en février 1968. Le MRPP sera le groupe le plus diffusé dans le milieu universitaire de Lisbonne, au moyen de ses sections Fédération des Etudiants Marxistes-Léninistes (FEML, Federação dos Estudantes MarxistasLeninistas) et Mouvement Populaire Anti-Colonial (MPAC, Movimento Popular AntiColonial). Il sera aussi très actif pendant la transition, quand il maintiendra une position antisystémique face aux gouvernements provisoires et de forte critique du PCP, principale cible 
Saldanha Sanches affirmait qu' "à Peniche, on était arrivé à une situation de totale réclusion en séparation 45 » et Fernando Rosas confirmait que :

«Oui, même à Peniche il y avait une séparation politique des prisonniers. À leur propre initiative d'ailleurs, parce qu'il y avait beaucoup d'incompatibilités dans l'attitude et la conduite des luttes au sein de la prison. Quand j'y étais, cette séparation existait déjà. Peu de temps auparavant, les détenus de différents groupes politiques en étaient presque venus aux poings, ce qui a conduit à la séparation par étage 46 ».

Ces dynamiques dans le microcosme du fort de Peniche reflétaient celles qui se manifestaient en dehors de la prison. Là, le conflit entre les différentes organisations de gauche et d'extrême gauche était en train de s'intensifier et entraînait une prolifération de groupes qui s'affrontaient entre eux. Par contre, tous ces groupes partageaient une dure critique contre le PCP, surtout à cause de sa stratégie considérée trop modérée, au point où il devint, à la fin du régime, l'une des principales cibles de la propagande de la nouvelle gauche, tout autant que le régime lui-même.

Le 18 décembre 1971, à l'âge de 27 ans, Sanches termina sa deuxième et plus longue détention. Comme nous l'avons vu, il a vécu une partie de ces dynamiques dans le milieu carcéral. Toutefois, on peut aisément imaginer que l'impact avec la réalité du monde extérieur ait été extrêmement fort, surtout si l'on considère combien l'accès à l'information était difficile en prison. Par exemple, selon les mots de Saul Nunes, les prisonniers de Peniche avaient su que Caetano avait remplacé Salazar seulement grâce aux prisonniers de droit commun. De plus, Nunes rappelle qu'il n'avait appris ce qui s'était passé à Paris en mai 68 qu'un an après. À sa sortie de prison, Sanches retourna à la Faculté de droit pour poursuivre ses études et il rencontra le MRPP, l'organisation maoïste la plus active et la plus enracinée dans ce milieu. C'est à cause de ce « deuxième engagement » qu'il sera encore arrêté deux fois avant la chute du régime et une fois pendant la transition démocratique : ce fut d'ailleurs la première arrestation politique de cette période.

de sa lutte, une position déjà mûrie pendant les dernières années du régime. Voir Accornero G., "La rivoluzione prima della rivoluzione ", in Accornero G. et Botti A. (eds.), Il Portogallo e la transizione alla democrazia, Numéro monographique de Storia e Problemi Contemporanei, 54, pp. 35-55 ; Accornero G., "Contentious Politics and Student Dissent in the Twilight of the Portuguese Dictatorship: Analysis of a Protest Cycle”, Democratization, DOI:10.1080/13510347.2012.674367 et Cardina M., A esquerda radical, Coimbra, Biblioteca Minima, 2010.

45. Entretien radiophonique par les journalistes Pedro Veiga, Maria dos Anjos Pinheiro et Esmeralda Serrano de José Luís Saldanha Sanches et Fernando Rosas, programme «Em tempo de mudança, a História do Século XX », transcription de Irineu Batista, Centro Documentação 25 de Abril, Université de Coimbra, 25 avril 1997.

46. Ibidem. 
Le 25 avril 1974, un coup d'État militaire préparé par le Mouvement de forces armés (Movimento das Forças Armadas - MFA) renverse le régime de l'Estado Novo. La transition portugaise vers la démocratie commence, caractérisée par une forte orientation à gauche en raison de l'importante influence du PCP sur le MFA et sur les gouvernements provisoires, mais aussi et surtout de l'explosion des mouvements sociaux. On caractérise d'ailleurs cette période de "processus révolutionnaire en cours " (Processo Revolucionário Em Curso - PREC), qui prend fin avec les épisodes du 25 novembre 1975, lorsque la tentative de coup d'État de militaires proches de la gauche radicale fut avortée par un contre-coup de militaires modérés, mettant fin à l'agitation sociale qui s'était intensifiée pendant «l'été chaud» (verão quente).

Le 25 avril 1974, Saldanha Sanches était détenu à la prison de Caxias et purgeait une peine de huit ans. Dans un entretien accordé en 1997 47, il affirme que le matin de cette journée, la vie dans la prison semblait se dérouler normalement. La seule « anomalie » semblait être que des prisonniers qui devaient aller au tribunal n'y étaient pas allés. Il raconte aussi « qu'il y avait un grand scepticisme sur la possibilité d'un coup d'État militaire. Cette hypothèse nous semblait un peu ridicule 48 ».

En outre, l'étrangeté de ce qui se passait en dehors de la prison était amplifiée par le fait que les visites et la lecture de journaux avaient été interdites à titre de punition à cause d'une lutte menée par les détenus ${ }^{49}$. La situation devint claire quand un lieutenant des parachutistes déclara qu'il y avait eu un coup d'État et que les prisonniers pouvaient sortir, après quoi les cellules furent ouvertes. Toutefois, des conditions furent tout de suite imposées : les prisonniers de la Ligue d'union et d'action révolutionnaire (Liga de União e Acção Revolucionária - LUAR ${ }^{50}$ ) ne pouvaient pas quitter la prison, à cause du type d'action dont ils étaient accusés, à savoir les vols, les agressions, etc. Les prisonniers, à l'initiative du PCP (et selon Sanches probablement à cause de la question de l’Action révolutionnaire armée - Acção Revolucionária

47. Ibidem.

48. Ibidem.

49. Comme décrit plus bas, dans la prison de Caxias il était normal que des luttes communes soient menées par les détenus qui se reconnaissaient dans les deux courants «prochinois » et «prosoviétique ». Cela peut s'expliquer par le fait que la prison de Caxias était plutôt une prison de passage, alors que dans celle de Peniche on pouvait passer beaucoup d'années, avec la conséquence que les frontières entre les différentes identités politiques devenaient très marquées et les clivages bien plus profonds.

50. La LUAR fut créée à Paris le 19 juin 1967 par un groupe hétérogène des dissidents du régime. L’idéologie de cette organisation n'était pas très définie : on y trouvait des socialistes aux républicains de droite, en passant par les catholiques progressistes et des militants qui, après la chute du régime deviendront dirigeants du Parti social démocrate, de centre-droit (PPD/PDS, Partido Social Democrata). Les militants de la LUAR étaient aussi accusés de cambriolages de banques, de vol de matériel militaire ou d'attentats contre les forces de l'ordre. 
Armada ${ }^{51}$ ), décidèrent qu'ils sortiraient seulement si tout le monde pouvait le faire. Comme l'indique Sanches, cette décision pouvait être prise à Caxias, où il y avait une coexistence plus ou moins pacifique entre tous les prisonniers, mais elle n'aurait pas été possible à Peniche, où, comme nous l'avons vu, il y avait eu une séparation radicale entre les détenus des différents courants. Finalement, le 26 avril, tous les prisonniers quittent la prison.

Selon Fernando Rosas, Sanches fut le premier « visage public » du MRPP après le 25 avril 1974. Cela semble en partie paradoxal, dans la mesure où il avait passé les dernières années du régime en prison. Il fut également le premier directeur du principal journal de cette organisation, Luta Popular, qui redevint légal. Comme les autres militants du MRPP, il demeure très critique face aux auteurs du coup d'État, et défend le fait que les masses populaires auraient dû mener leur propre révolution et ne devraient pas «sortir dans la rue » pour soutenir une révolution bourgeoise. Il est intéressant de lire à ce propos le premier document diffusé par le MRPP après le coup, qui soutient que l'action de «l'armée colonial-fasciste » se limitait à une «lutte entre les deux groupes de la classe dirigeante », et déclare que « quel que soit le résultat concret de cette lutte intestine et réactionnaire [...] ni le peuple, en général, ni la classe ouvrière, en particulier, ne devait alimenter des illusions 52 ».

En tant que directeur de Luta Popular, Sanches reste, pendant les premiers mois de la révolution, très hostile au MFA, comme en témoigne l'article Nem mais um embarque ${ }^{53}$, où il incite les soldats à la désertion en emportant leurs armes. La conséquence la plus immédiate de cet article fut son arrestation, la première arrestation politique sous le nouveau régime. Il fut arrêté le 7 juin 1974 et demeura emprisonné pendant onze jours.

Toutefois, au cours de ces mois post-coup, Sanches eut aussi la possibilité de reprendre des activités interrompues à cause de sa longue détention pendant le régime. Il commence aussi à développer une critique du MRPP luimême, qui se manifeste très clairement à la fin du PREC, en décembre 1975, quand il publie le petit livre $O M R P P$ - instrumento da contra-revolução ${ }^{54}$, qui marque le moment de sa sortie du militantisme. D'après la femme de Saldanha Sanches, Maria José Morgado ${ }^{55}$, c'est aussi à partir de ce moment-là

51. L'ARA avait été créée par le PCP, normalement opposé à la lutte armée, en 1966 dans une logique de compétition à gauche par la quelle il se sentait menacé de perdre de l'espace politique par les nouvelles organisations plus radicales. Son activité, qui consistait surtout à voler du matériel militaire et à faire des sabotages, ne commença qu'en 1970 et cessa en 1973.

52. Comité Lenine, Organisme Central du MRPP, "Comunicado ao Povo Português do MRPP », 25 Avril 1974.

53. Luta Popular, 30 mai 1974, p. 1.

54. Saldanha Sanches J. L., O MRPP: instrumento da contra-revolução, Lisboa, Ulmeiro, 1975.

55. Entretien avec Maria José Morgado, réalisé dans son bureau à la « cité judiciaire », le 28 juillet 2010. Maria José Morgado était aussi militante du MRPP. Elle rencontra Saldanha Sanches en 1971 à la Faculté de droit de Lisbonne où elle étudiait aussi. Elle quitta le parti en même temps que son mari en 1975. Aujourd'hui, elle est magistrate du Ministère public portugais. Elle est 
qu'il commence à faire des projets familiaux - avec la naissance de leur enfant en 1976. Il reprend ses études, qu'il termine en 1978, et devient assistant à la Faculté de Droit de Lisbonne.

Les histoires des exilés politiques qui ont eu la possibilité de rentrer au pays après le 25 avril 1974 sont assez similaires. La chute du régime leur a permis de revenir et d'entreprendre de nouveaux projets professionnels et personnels et de tisser de nouveaux réseaux sociaux. Certains ont même cessé de s'impliquer directement dans des activités politiques. Dans le même temps, les ressources acquises par la militance, reconverties de différentes façons, constituent à leur tour des éléments fondamentaux dans la «nouvelles vie » des exmilitants ${ }^{56}$. Comme le souligne Catherine Leclercq, « les défections [...] gagnent à être saisies en relation avec l'éventail des possibles noués dans les biographies individuelles 57 ». Toutes ces trajectoires sur un plan individuel forment le processus de désengagement que certains chercheurs ont observé, à une échelle plus large, comme une caractéristique de la vie sociale et politique du Portugal après le verão quente, c'est-à-dire au terme du processus révolutionnaire à la fin de 197558 .

Sanches fut l'un des militants les plus importants de l'agitation étudiante et de la résistance anti-Estado Novo dans la dernière décennie de la dictature portugaise, aussi bien avec le PCP qu'avec l'extrême gauche. Il joua ce rôle dans un état d'absence physique presque constante, puisqu'il passa plus de 7 ans et demi en prison au total. Entre le moment où il est apparu comme leader étudiant en 1964 et le 25 avril 1974, il n’a pu développer son activité politique en-dehors de la prison que durant deux années et demi, pendant lesquelles il faut aussi considérer les périodes passées dans la clandestinité. Il joua néanmoins un rôle mobilisateur aussi et surtout par son absence, et, en un sens, son emprisonnement fut l'un des arguments les plus forts de la contestation contre la répression du régime au cours de ses dernières années.

très connue pour sa lutte contre la corruption au Portugal.

56. Pour une analyse de l'engagement politique du point de vue de l'acquisition de ressources et de l'apprentissage de savoirs et savoir-faire, militants ou non, voir Leclercq C., "Engagement et construction de soi. La carrière d'émancipation d'un permanent communiste ", Sociétés contemporaines, 2011, 4, pp. 127-149. Selon elle, ces mêmes ressources peuvent être à la base du désengagement lui-même : "Tout se passe comme si, paradoxalement, les investissements libidinaux offerts par l'institution étaient en permanence susceptibles de se retourner contre elle en rendant ses membres à la fois plus confiants et plus exigeants », Ibidem, p. 34.

57. Leclercq C., " "Raisons de sortir". Les militants du Parti communiste français", in Fillieule O. (ed.), Le désengagement militant, Paris, Belin, 2005, p. 154.

58. Entre autres, de Sousa Santos B., O Estado e a sociedade em Portugal (1974-1988), Porto, Afrontamento, 1990; Braga da Cruz M., Instituições políticas e processos sociais, Venda Nova, Bertrand, 1995 ; Schmitter P., Portugal: do autoritarismo à democracia, Lisboa, Imprensa de Ciências Sociais, 1999 ; Palácios Cerezales D., O poder caiu na rua. Crise de Estado e Acções colectivas na Revolução Portuguesa, 1975-1975, Lisboa, Imprensa de Ciências Sociais, 2003 ; Wiarda H. J. (ed.), Development on the Periphery: Democratic Transitions in Southern and Eastern Europe, Lanham, Rowman \& Littlefield, 2006. 


\section{Conclusion}

Comme le souligne José Maria Maravall à propos du cas de la dissidence estudiantine et ouvrière dans les dernières périodes du régime franquiste,

«Il faut prendre en considération trois conditions dans le processus de conversion idéologique non-conformiste : l'accessibilité aux idéologies de cette nature, la conversion à ces idéologies et la concrétisation des opinions sous forme d'action politique. Ces trois phases successives sont déterminées par les conditions politiques existantes qui peuvent agir comme restriction au radicalisme. Ces restrictions peuvent être normatives ou non normatives (matérielles), préventives ou répressives. De ce point de vue, devenir dissident politique dans un contexte non démocratique peut être interprété comme une forme de “déviance” sociale 59 ».

Cela signifie que devenir militant dans un contexte autoritaire comporte des coûts beaucoup plus élevés que dans un régime démocratique. La répression fait partie de ces coûts. Il est question ici de la répression « directe », celle qui caractérise le contrôle de la rue, la violence contre les manifestants, l'emprisonnement des dissidents, l'absence de garanties pour les détenus, la torture ou encore les punitions dans les prisons politiques. Mais il y a aussi la répression plus « indirecte », celle qui empêche les militants de l'opposition de trouver un travail, de poursuivre leurs études, d'avoir des relations sociales. Toutefois, comme nous avons essayé de le montrer, ces coûts élevés pour devenir « dissident » rendent aussi la sortie de ce militantisme très « chère » et expliquent aussi les liens sociaux très forts à la base du militantisme. Dans ce contexte, bien souvent, la seule sortie possible (si on omet les rares cas des dissidents qui plus ou moins volontairement sont devenus des collaborateurs du régime) est le passage d'un groupe à un autre. Enfin, on peut affirmer qu'un régime autoritaire, comme dans le cas du régime portugais, peut avoir pour effet d'absolutiser le militantisme politique et de rendre le désengagement très difficile. On peut voir ce mécanisme en action à partir de différents points de vue. D’abord, du point de vue identitaire. Le coût psychique très élevé à payer pour assumer l'identité d'adversaire, de « déviants » (pour reprendre l'expression de Maravall) se reflète dans le coût très élevé lui aussi du renoncement à cette identité. Ensuite, du point de vue matériel. En éliminant la plupart des issues possibles pour sortir de l'engagement, le régime rend le désengagement très difficile à cause de l'impossibilité d'une éventuelle reconversion. Enfin, et en liaison avec le point précédent, la chute du régime permet, pour ceux qui veulent accepter les nouvelles règles du jeu, de sortir de la clandestinité.

59. Maravall J.M., Dictadura y disentimiento politico : obreros y estudiantes bajo el franquismo, Madrid, Alfaguara, 1978, p. 191. 
Si on considère, en suivant Donatella della Porta, que «dans la clandestinité, les organisations deviennent de plus en plus compartimentées et fermées sur l'extérieur [...] et l'idéologie a aussi tendance à devenir de plus en plus obscure pour ceux qui n'en font pas partie et devient un instrument de consommation interne» 60 , on voit bien que quitter la clandestinité (cette dernière étant définitivement liée à la configuration institutionnelle) peut signifier aussi quitter le militantisme lui-même. Dans ce cadre, on peut aussi voir le mécanisme que Leclercq dénomme "déracinement ", selon lequel «la légitimité des militants formés dans un état antérieur de l'institution est remise en question [...]. Les effets du changement institutionnel peuvent donc prendre différentes formes suivant les profils socioprofessionnels et générationnels, les histoires personnelles et les contextes institutionnels » 61 . Leclercq a notamment repéré ce mécanisme qui permet de " penser l'articulation entre les évolutions institutionnelles et les transformations individuelles » 62 dans le cas d'un changement institutionnel au niveau organisationnel, en analysant les défections au sein du Parti Communiste Français (PCF) au moment où il souffre d'une recomposition et où sa position change dans le champ partisan. Dans le cas de notre étude, ce mécanisme de restructuration identitaire semble s'opérer de façon bien plus forte, puisque le « macro-déterminant » est bien plus radical : il s'agit d'un véritable changement de régime.

60. Della Porta D., « Mouvements sociaux et violence politique », in Crettiez X. et Mucchielli L. (eds.), Les Violences politiques en Europe. Un état des lieux, Paris, La Découverte, 2010, p. 282.

61. Leclercq C., Histoires d"'ex". Une approche socio-biographique du désengagement des militants du Parti communiste français, thèse de doctorat en Science politique. Sociologie politique et politiques publiques, Paris, Institut d'études politiques, 2008, p.980.

62. Ibidem. 